\title{
Rapid Newcastle Disease Virus Detection Based on Loop-Mediated Isothermal Amplification and Optomagnetic Readout
}

Tian, Bo; Ma, Jing; Zardán Gómez de la Torre, Teresa; Bálint, Ádám; Donolato, Marco; Hansen, Mikkel Fougt; Svedlindh, Peter; Strömberg, Mattias

\section{Published in:}

Sensors

Link to article, DOI:

$10.1021 /$ acssensors.6b00379

Publication date:

2016

Document Version

Publisher's PDF, also known as Version of record

Link back to DTU Orbit

Citation (APA):

Tian, B., Ma, J., Zardán Gómez de la Torre, T., Bálint, Á., Donolato, M., Hansen, M. F., Svedlindh, P., \& Strömberg, M. (2016). Rapid Newcastle Disease Virus Detection Based on Loop-Mediated Isothermal Amplification and Optomagnetic Readout. Sensors, 1(10), 1228-1234. https://doi.org/10.1021/acssensors.6b00379

\section{General rights}

Copyright and moral rights for the publications made accessible in the public portal are retained by the authors and/or other copyright owners and it is a condition of accessing publications that users recognise and abide by the legal requirements associated with these rights.

- Users may download and print one copy of any publication from the public portal for the purpose of private study or research.

- You may not further distribute the material or use it for any profit-making activity or commercial gain

- You may freely distribute the URL identifying the publication in the public portal 


\title{
Rapid Newcastle Disease Virus Detection Based on Loop-Mediated Isothermal Amplification and Optomagnetic Readout
}

\author{
Bo Tian, ${ }^{\dagger}$ Jing Ma, ${ }^{\ddagger}$ Teresa Zardán Gómez de la Torre, ${ }^{\dagger}$ Ádám Bálint, ${ }^{\#}$ Marco Donolato,
}

Mikkel Fougt Hansen, $"$ Peter Svedlindh, ${ }^{\dagger}$ and Mattias Strömberg*, ${ }^{\dagger}$

\author{
${ }^{\dagger}$ Department of Engineering Sciences, Uppsala University, The Ångström Laboratory, Box 534, SE-751 21 Uppsala, Sweden \\ ${ }^{\ddagger}$ Department of Immunology, Genetics and Pathology, Uppsala University, The Rudbeck Laboratory, SE-751 85 Uppsala, Sweden \\ ${ }^{\#}$ National Food Chain Safety Office, Veterinary Diagnostic Directorate, Tábornok u. 2., H-1143 Budapest, Hungary \\ ${ }^{\S}$ BluSense Diagnostics, Fruebjergvej 3, 2100 Copenhagen, Denmark \\ "Department of Micro- and Nanotechnology, Technical University of Denmark, DTU Nanotech, Building 345 East, DK-2800 \\ Kongens Lyngby, Denmark
}

\section{Supporting Information}

\begin{abstract}
Rapid and sensitive diagnostic methods based on isothermal amplification are ideal substitutes for PCR in out-of-lab settings. However, there are bottlenecks in terms of establishing low-cost and user-friendly readout methods for isothermal amplification schemes. Combining the high amplification efficiency of loop-mediated isothermal amplification (LAMP) with an optomagnetic nanoparticle-based readout system, we demonstrate ultrasensitive and rapid detection of Newcastle disease virus RNA. Biotinylated amplicons of LAMP and reverse transcription LAMP (RT-

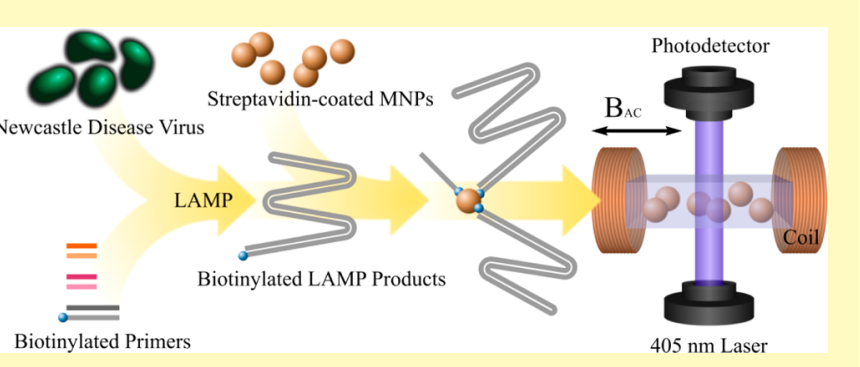
LAMP) bind to streptavidin-coated magnetic nanoparticles (MNPs) resulting in a dramatical increase in the hydrodynamic size of the MNPs. This increase was measured by an optomagnetic readout system and provided quantitative information on the amount of LAMP target sequence. Our assay resulted in a limit of detection of $10 \mathrm{aM}$ of target sequence with a total assay time of $30 \mathrm{~min}$. The assay has also been tested on clinical samples (vaccine and tissue specimens) with a performance comparable to realtime RT-PCR. By changing the LAMP primers, this strategy can serve as a general method for the detection of other DNA/RNA targets with high specificity and sensitivity.
\end{abstract}

KEYWORDS: out-of-lab diagnostics, Newcastle disease virus, loop-mediated isothermal amplification, optomagnetic bioassay, magnetic nanoparticles

ince emerging infectious diseases of free-living and
domestic animals have been a serious problem for both
human and animal health during the past decades, early
diagnosis and efficient monitoring of pathogen spread are
therefore of crucial importance. ${ }^{1}$ Newcastle disease (ND),
together with highly pathogenic avian influenza, are regarded as
the two most significant diseases of poultry as well as of other
birds throughout the world. ${ }^{2}$ Caused by Newcastle disease virus
(NDV), the ND outbreaks have flock mortality rates up to
$100 \%$ in fully susceptible chickens, and can spread rapidly,
resulting in severe economic losses. ${ }^{3,4}$ While virus isolation in
embryonated eggs remains the gold standard for NDV
detection, molecular diagnostics, especially real-time reverse
transcription PCR (real-time RT-PCR), are more commonly
used because of their reliability. ${ }^{5,6}$ However, real-time RT-PCR
based methods require high-precision thermal cycling equip-
ment, expensive optical detection of PCR amplification
products (e.g., fluorescence readers), and complicated oper-
ation requiring specially trained personnel. This limits the potential for RT-PCR for rapid and cost-effective on-site epizootic monitoring especially in developing countries.

Loop-mediated isothermal amplification (LAMP), a molecular tool that offers isothermal enzymatic amplification conditions, has the potential to revolutionize molecular diagnostics by its high specificity, high amplification efficiency, avoidance of sophisticated thermal cycling devices, low cost, and the ability of being applied to nondenatured DNA samples. ${ }^{7,8}$ Although a well-designed LAMP assay can generate more than $10^{9}$ amplicons within $1 \mathrm{~h}$, the performance of LAMP highly depends on the particular readout method chosen for analyzing the amplification products and/or byproducts. ${ }^{9,10}$ LAMP reactions can easily be monitored by the naked eye owing to the precipitation of $\mathrm{Mg}_{2} \mathrm{P}_{2} \mathrm{O}_{7}$, a byproduct produced in a successful LAMP reaction. ${ }^{11}$ However, this naked-eye measurement only provides a qualitative result with limited

Received: June 10, 2016

Accepted: September 19, 2016

Published: September 19, 2016 
sensitivity. ${ }^{10,12}$ Although DNA-binding dyes such as ethidium bromide and other colorimetric indicators such as calcein were employed to obtain a higher sensitivity than that obtained by turbidity alone, sensitive and rapid monitoring methods still remain a prime objective. ${ }^{10}$ In recent years, several sensor technologies have been reported as detection methods of LAMP amplification products, including electrochemical sensors, ${ }^{13-16}$ surface plasmon resonance (SPR) sensors, ${ }^{17,18}$ resistive pulse sensors, ${ }^{19}$ quartz crystal microbalance (QCM) devices, ${ }^{20}$ field effect sensors, ${ }^{21}$ giant magnetoresistive (GMR) sensor technologies, ${ }^{22}$ and surface enhanced Raman spectroscopy (SERS). ${ }^{23}$ Most of those reported sensors are surfacebased. Although utilization of those surface-based sensor devices has significantly improved the sensitivity of LAMP based methods, their total assay time is limited by the diffusion of LAMP products/byproducts to the sensor surface (10-100 $\mu \mathrm{m}$ movement in the minutes-to-hours time scale). ${ }^{24,25}$ In contrast to the surface-based sensors, volumetric sensors measure the entire detection volume and acquire the ensemble average signal without the need of an immobilization/removal process, thus enabling rapid, low-cost, and user-friendly detection. $^{25}$

Due to the properties such as superparamagnetism and biocompatibility, as well as the low background magnetic signal of biological samples, magnetic nanoparticles (MNPs) are widely used in bioanalytical methods. However, compared to optical detectors, magnetic readout sensors (e.g., nuclear magnetic resonance systems) are usually bulky, sophisticated, and expensive. To analyze the magnetic signal/property of MNPs in a more convenient and cost-effective way, optical sensors have been combined with magnetic actuators to detect the periodic movements of magnetic chains/aggregates induced by a magnetic field and by the presence of analytes. ${ }^{26-29}$

Optomagnetic sensors, which measure the AC magnetic excitation field-induced modulation of the transmission signal from samples with MNPs, have been considered as a rapid and low-cost volumetric MNP detection system. The $405 \mathrm{~nm}$ laserbased optomagnetic readout system, first reported in 2014 by Donolato et al., ${ }^{30}$ has been employed for the detection of several kinds of biomolecules and pathogens ${ }^{31-33}$ including DNA sequences. ${ }^{34,35}$ Previous DNA detections describing the optomagnetic readout based on detection of rolling circle amplification (RCA) products have reached a limited sensitivity on the order of hundreds of femtomolar due to lack of amplification efficiency of RCA. In addition, RCA-based analysis procedures have rather complex and time-consuming protocols including several steps such as padlock probe ligation, rolling circle amplification, MNP labeling, and read-out, resulting in a total analysis time of about $1.5 \mathrm{~h}^{34,35}$

LAMP has been reported for NDV detection with a sensitivity of $96.8 \%$ and a limit of detection (LOD) of 75 $\mathrm{fM}$; visual inspection and electrophoresis analysis were used and the total assay times were around $50 \mathrm{~min}$ and more than 120 min, respectively. ${ }^{36,37}$ The sensitivity is limited by the lack of appropriate readout method for the LAMP reaction. Here, we present a rapid and simple NDV detection method combining LAMP and an optomagnetic nanoparticle-based readout. Biotinylated LAMP amplicons resulting from the use of biotinylated primers were attached to streptavidin-coated MNPs and subsequently measured by a $405 \mathrm{~nm}$ laser-based optomagnetic setup. An increased concentration of LAMP products resulted in a peak shift to lower frequencies for the measured optomagnetic response. To the best of our knowledge, this is the first time an optomagnetic sensor is reported for direct virus detection. This novel detection method, which provides an LOD of $10 \mathrm{aM}$ with a total assay time of $30 \mathrm{~min}$, not only benefits epizootic monitoring of NDV, but can also be adapted to detect other RNA viruses.

\section{EXPERIMENTAL SECTION}

Clinical Samples and RNA Extraction. A total of 29 clinical NDV-infected samples were collected by Prophyl Ltd. (Mohács, Hungary) and prepared by the National Food Chain Safety Office Veterinary Diagnostic Directorate (Budapest, Hungary). Tissue samples were suspended in phosphate buffered saline (PBS) at a concentration of $0.5 \mathrm{~g} / \mathrm{mL}$ and homogenized in TissueLyser (QIAGEN, Heiden, Germany) at $30 \mathrm{~Hz}$ for $2 \mathrm{~min}$, followed by centrifugation at $5000 \mathrm{~g}$ for $10 \mathrm{~min}$. Supernatants of $100 \mu \mathrm{L}$ were complemented with $40 \mu \mathrm{L}$ PBS and extracted with the QiaAmp Viral RNA Mini Kit (QIAGEN, Heiden, Germany). The extracted RNA samples were eluted in $100 \mu \mathrm{L}$ elution buffer and stored at $-80{ }^{\circ} \mathrm{C}$. Real-time RT-PCR analysis was performed by the National Food Chain Safety Office Veterinary Diagnostic Directorate as a standard biomedical assay for comparison.

Primer Design. A conserved region of NDV strain Herts/33/NS fusion protein (F) gene (GenBank accession number: AY117024) was chosen as the target gene for LAMP and RT-LAMP primer design. An inner primer pair (FIP/BIP), an outer primer pair (F3/B3), and a loop primer pair $(\mathrm{LF} / \mathrm{LB})$ were designed using Primer Explorer $\mathrm{v} 4$ (https://primerexplorer.jp/e/), as shown in Table S1 and SI 2. Inner primer FIP was labeled with a $5^{\prime}$ biotin group. The six primers as well as the $230 \mathrm{bp}$ target sequence were synthesized by Integrated DNA Technologies (Coralville, USA) and used for both synthetic DNA detection and clinical RNA sample detection.

LAMP Reaction, MNP Labeling of Amplicons, and Dynamic Light Scattering Characterization of LAMP Amplicons and Amplicon-MNPs. A $50 \mu \mathrm{L}$ reaction mixture contained $0.2 \mu \mathrm{M}$ of outer primers, $0.4 \mu \mathrm{M}$ of loop primers, different concentrations of inner primer $(0.2,0.4,0.8$, or $1.6 \mu \mathrm{M}), 1 \times$ isothermal amplification buffer II (containing $2 \mathrm{mM}$ of $\mathrm{MgSO}_{4}$, New England Biolabs, Ipswich, UK), $6 \mathrm{mM}$ of $\mathrm{MgSO}_{4}$ ( $8 \mathrm{mM}$ in total, New England Biolabs), $16 \mathrm{U}$ of Bst 3.0 polymerase (New England Biolabs), $1.4 \mathrm{mM}$ dNTPs (Thermo Fisher Scientific, Waltham, USA), and $20 \mu \mathrm{L}$ of analyte solution (containing synthetic DNA or RNA from clinical sample). For the LAMP reaction, the mixture was incubated at various temperatures for $15 \mathrm{~min}$. After amplification, the $\mathrm{Mg}_{2} \mathrm{P}_{2} \mathrm{O}_{7}$ precipitate was separated from the amplicons by a few seconds of centrifugation. The supernatant was mixed with $200 \mu \mathrm{g} / \mathrm{mL}$ of streptavidin-coated 100 nm MNPs (Micromod, Rostock, Germany) at 1:1 volumetric ratio followed by standing at room temperature for $10 \mathrm{~min}$. The centrifugation was not necessary if the peak frequency calculation was used for the judgment of results. For size characterization of the amplicon-MNP samples, the amplicon-MNPs were separated by a magnetic stand, resuspended in PBS, and measured using a dynamic light scattering (DLS) instrument (Malvern Zetasizer Nano ZS, Malvern Instruments, Malvern, UK) equipped by a $633 \mathrm{~nm} \mathrm{He}-\mathrm{Ne}$ laser and operating at an angle of $173^{\circ}$ at $25^{\circ} \mathrm{C}$.

Optomagnetic Measurement Setup and Readout of Amplicon-MNP Samples. A detailed description of the setup and underlying theory is provided in Supporting Information, section S1. The output from one measurement (used for target quantification) is the total intensity of transmitted laser light, $V_{0}$, the in-phase, $V_{2}^{\prime}$, and the out-of-phase, $V_{2}^{\prime \prime}$, components of the complex second harmonic signal of the transmitted light. $V_{2}^{\prime}$ is normalized with respect to the simultaneously measured value of $V_{0}$ to compensate for the variations in laser light intensity, particle concentration, and cuvette reflection/ absorption.

For optomagnetic measurements, $65 \mu \mathrm{L}$ of the amplicon-MNPs were transferred into a disposable UV-transparent cuvette (REF 67.758.001, SARSTEDT, Nümbrecht, Germany). Measurements of $V_{2}^{\prime}$ and $V_{0}$ at room temperature were performed in the frequency range of $10-1000 \mathrm{~Hz}$, using an AC magnetic excitation field amplitude of 2.6 


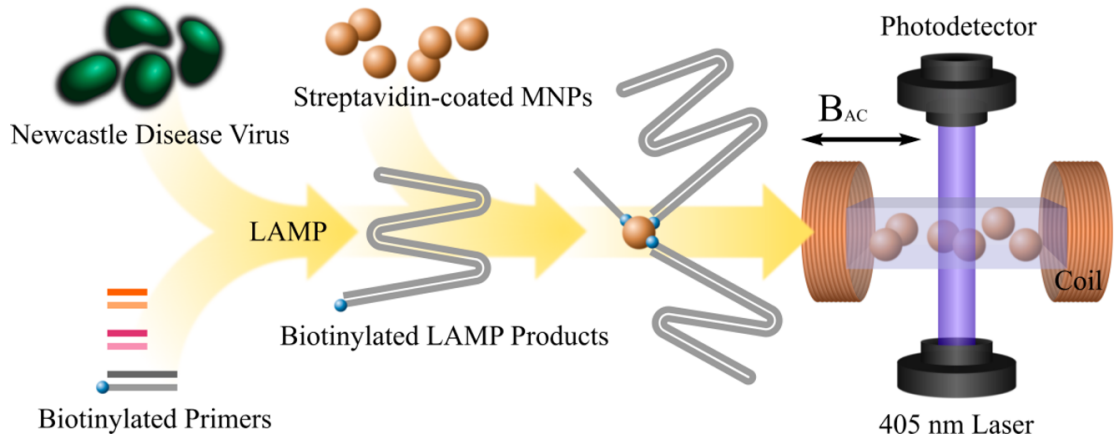

Figure 1. Schematic illustration of LAMP-optomagnetic NDV detection. The assay includes three independent steps (from left to right): LAMP reaction, labeling, and measurement. LAMP reaction: target sequences are amplified by LAMP and generate biotinylated amplicons. Labeling: streptavidin-coated MNPs are mixed with the amplicons followed by incubation. Measurement: the amplicon-MNPs are transferred into a cuvette and measured using the optomagnetic readout system.
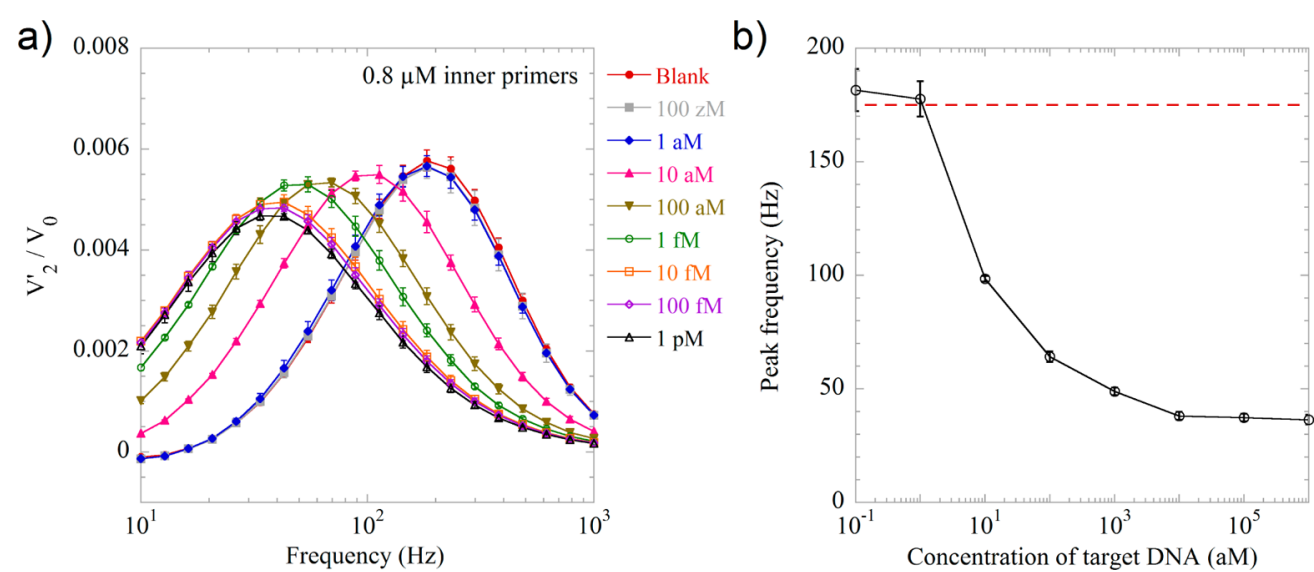

Figure 2. Synthetic target DNA detection using an optimum inner primer concentration of $0.8 \mu \mathrm{M}$. (a) $V_{2}^{\prime} / V_{0}$ spectra for the indicated concentrations of synthetic DNA target. (b) Dose-response curve for synthetic target DNA detection. Error bars indicate one standard deviation based on three independent measurements. The cutoff value is indicated by the horizontal line.

$\mathrm{mT}$ applied perpendicular to the propagation direction of the laser light. The optical path through the cuvette was $10 \mathrm{~mm}$.

\section{RESULTS AND DISCUSSION}

Optomagnetic Detection and Setup. The LAMPoptomagnetic detection of NDV is illustrated in Figure 1. Target RNA extracted from NDV (or synthetic target DNA) is mixed with LAMP reagents including biotinylated inner primers and amplified by LAMP. After reaction, the amplicons are captured by streptavidin-coated MNPs through biotinstreptavidin interaction and subsequently measured by an optomagnetic sensor system including a $405 \mathrm{~nm}$ laser, a photodetector for measuring the intensity of transmitted light and two electromagnets for creating an alternating magnetic excitation field. The binding of amplicons increases the hydrodynamic volume of MNPs, resulting in a change in the rotational dynamics of MNPs in the suspension when actuated by the external magnetic field. The amount of LAMP amplicons captured by the MNPs is quantified by measuring the AC magnetic field-induced modulation of the light transmitted through the sample in terms of the in-phase component of the complex second harmonic signal of the transmitted light, $V_{2}^{\prime}$.

LAMP Assay Optimization. To find the optimal reaction temperature and primer concentration, agarose gel electrophoresis analysis was employed where synthetic target DNA (Table S1, a conserved region of NDV strain Herts/33/NS fusion protein gene, GenBank accession number: AY117024) at a concentration of $1 \mathrm{pM}$ was used as analyte. The optimum reaction temperature was found to be $67{ }^{\circ} \mathrm{C}$ for $15 \mathrm{~min}$ of amplification (Figure S3a). The composition of the primer mixture was optimized by testing different ratios of inner and outer primers (Figure S3b). The reaction efficiency was improved for higher concentrations of inner primers. However, more inner primers resulted in more free biotinylated primers and short biotinylated amplicons in the suspension after amplification, which could block the MNPs, leading to a lower Brownian relaxation frequency shift. To find an optimum inner primer concentration that balanced the reaction efficiency and the blocking effect caused by the excess of biotinylated primers, different primer concentrations were evaluated by detecting a range of concentrations of synthetic target DNA.

Amplification time is another critical parameter for LAMP. A longer reaction time provides stronger positive results, which always means a higher sensitivity especially for naked-eye judgment. However, due to the end-point detection format and the high amplification efficiency of LAMP, signals of positive samples easily reach the saturation level and are then not useful for quantification. Although a longer reaction time can produce longer amplicons leading to larger changes in hydrodynamic volume of MNPs (Figure S4), the dynamic detection range becomes narrower. Moreover, longer reaction time gives a higher risk of false-positive results caused by carryover contamination, which is common in LAMP works, especially when the detection procedure requires opening of the LAMP 
reaction tube. ${ }^{8}$ After optimization (data not shown), we chose an amplification time of 15 min to achieve a sensitive and rapid detection system without limiting the dynamic detection range.

Detection of Synthetic Target DNA. Since Bst 3.0 polymerase is able to amplify both RNA and DNA sequences under identical conditions, synthetic NDV DNA was chosen as a model to find the optimum primer concentration and to determine the LOD of the LAMP-optomagnetic detection assay. The response vs target concentration, $c$, for different concentrations of inner primers $(0.2,0.4,0.8$, and $1.6 \mu \mathrm{M})$ is presented in Figure S5. We found that the $V_{2}^{\prime} / V_{0}$ peak shifted to lower frequency with increasing $c$ whereas the peak amplitude was nearly constant. This observation implies that the LAMP amplicons bind to the MNPs but do not cause crosslinking of MNPs. Thus, a higher target concentration gives a denser layer of LAMP amplicons on the MNPs. This enabled detection based on shifts of the peak position, $f_{\mathrm{p}}$, rather than on the reduction of the peak amplitude at a fixed frequency used in previous studies. ${ }^{30,38-40}$ Depending on the primer concentration, we observed $f_{\mathrm{p}}$ shifts from $183 \mathrm{~Hz}$ to a value in the range of $30-160 \mathrm{~Hz}$. We found that an inner primer concentration of $0.8 \mu \mathrm{M}$ was optimal since this value gave the largest $f_{\mathrm{p}}$ shift for low $c$ values (Figure S5). The spectra obtained for this concentration of inner primers are shown in Figure 2a. The dose-response curve of $f_{\mathrm{p}}$ vs $c$ is shown in Figure $2 \mathrm{~b}$ for triplicate measurements. The cutoff value was calculated as the average signal of the blank control samples minus three standard deviations, and the LOD was defined as the lowest $c$ whose value is below the cutoff. Figure S6 shows the dose-response curves obtained for all investigated inner primer concentrations.

The presented analysis in terms of $f_{\mathrm{p}}$ has the advantage that it is insensitive to a variation in the transmitted light intensity, e.g., due to formation and precipitation of $\mathrm{Mg}_{2} \mathrm{P}_{2} \mathrm{O}_{7}$ during the reaction (a scanning electron microscope image of $\mathrm{Mg}_{2} \mathrm{P}_{2} \mathrm{O}_{7}$ precipitation is shown in Figure S7), variation in the sample color and variations between the cuvettes. The characteristic frequency for Brownian relaxation is given by $f_{\mathrm{B}}=k_{\mathrm{B}} T$ / $\left(6 \pi \eta V_{\mathrm{h}}\right)$, where $k_{\mathrm{B}} T$ is the thermal energy, $\eta$ is the viscosity, and $V_{\mathrm{h}}$ is the mean MNP hydrodynamic volume. It should be stressed that the position of a peak in Figure 2a and Figure S5 is related to the mean Brownian relaxation frequency for the population of MNPs with LAMP products bound to the surface, but not equal to it (see Supporting Information, Section S1). For this analysis approach, we found that the intraassay coefficient of variation (CV) ranged from $2 \%$ to $6 \%$. The $0.8 \mu \mathrm{M}$ inner primer concentration produced the largest $f_{\mathrm{p}}$ shifts (down to $f_{\mathrm{p}}=36 \mathrm{~Hz}$ ) and resulted in an LOD of $10 \mathrm{aM}$ and a dynamic detection range of approximately 3 orders of magnitude with a total assay time of $30 \mathrm{~min}$. Owing to the enzyme evolution, the Bst 3.0 polymerase provides much faster reaction kinetics compared to the Bst polymerase used in the previously reported LAMP-based methods. ${ }^{36,37}$ In addition, our analysis procedure is about 4 orders of magnitude more sensitive than previously reported LAMP based NDV detection assays. ${ }^{36}$ An LOD of $10 \mathrm{aM}$ in a $20 \mu \mathrm{L}$ sample volume approximately corresponds to 6 copies $/ \mu \mathrm{L}$ (120 copies/ reaction), which is comparable to state-of-the-art LAMP based biosensors (Table 1). The sensitivity of the presented method is also comparable to real-time RT-PCR (20-200 copies for NDV $)^{41-44}$ and about 100 times better than turbidity-based naked eye judgment (Figure S8).
Table 1. Sensitivity and Assay Time Comparison of Representative LAMP-Based Biosensors

\begin{tabular}{llrl}
\multicolumn{1}{c}{ detection method } & \multicolumn{1}{c}{ LOD } & $\begin{array}{c}\text { assay time } \\
\text { (min) }\end{array}$ & refs \\
Electrochemical sensor $^{b}$ & $10^{6}$ copies $/ \mu \mathrm{L}$ & $\sim 120$ & 13 \\
Electrochemical sensor $^{c}$ & 0.8 copies $/ \mu \mathrm{L}$ & 50 & 14 \\
Electrochemical sensor $^{b}$ & $100 \mathrm{CFU} / \mathrm{mL}$ & 40 & 15 \\
Electrochemical sensor $^{b}$ & $17 \mathrm{pg} / \mathrm{mL}$ & 70 & 16 \\
SPR $^{c}$ & $2 \mathrm{fg} / \mathrm{mL}$ & 25 & 17 \\
SPR $^{b}$ & $10 \mathrm{copies} / \mu \mathrm{L}$ & $>60$ & 18 \\
Resistive pulse sensor $^{b}$ & $500 \mathrm{copies} / \mu \mathrm{L}$ & $\sim 120$ & 19 \\
QCM $^{c}$ & $100 \mathrm{copies}$ & 30 & 20 \\
Field effect sensor $^{c}$ & $10^{8} \mathrm{copies}$ & 50 & 21 \\
GMR $^{b}$ & $1 \mathrm{copies} / \mu \mathrm{L}^{a}$ & 60 & 22 \\
SERS $^{b}$ & $66 \mathrm{CFU} / \mathrm{mL}$ & $>65$ & 23 \\
Optomagnetic sensor $^{b}$ & $6 \mathrm{copies} / \mu \mathrm{L}$ & 30 & Current work
\end{tabular}

${ }^{a}$ Concentration of sample after pretreatment and enrichment is listed here for comparison. ${ }^{b}$ End-point measurement format. ${ }^{c}$ Real-time measurement format.

Although the performed analysis in terms of $f_{\mathrm{p}}$ has many advantages, it is currently available only in the end-point detection format, since it takes about $2 \mathrm{~min}$ to record a spectrum for calculating $f_{\mathrm{p}}$. Another reason for choosing endpoint measurements is the $\mathrm{Mg}_{2} \mathrm{P}_{2} \mathrm{O}_{7}$ precipitation, which forms during the LAMP reaction and strongly influences the size increase of MNPs. Therefore, the size increase of MNPs can be attributed not only to the binding of amplicons to MNPs. For the end-point detection format, the dynamic detection range is limited and the signals from strong positive samples will be saturated and therefore not useful for quantification. To achieve a better quantification of strong positive samples, we can (1) perform serial dilutions before measurement, or (2) adjust the LAMP reaction time to rebalance the dynamic detection range with the sensitivity. The LAMP-Optomag detection system has the potential to be performed in a real-time format by running the LAMP reaction in the presence of streptavidin-MNPs. In the real-time format, the deposition of $\mathrm{Mg}_{2} \mathrm{P}_{2} \mathrm{O}_{7}$ precipitation on the surface of MNPs further increases the hydrodynamic size of MNPs, and the spectra can be recorded within $1 \mathrm{~min}$ when a narrower frequency range is monitored.

High concentrations of inner primers can accelerate the LAMP reaction and thus provide more amplicons, which can be seen in the gel electrophoresis photograph in Figure S3b. However, according to the data from the supplier, a final concentration of $100 \mu \mathrm{g} / \mathrm{mL}$ streptavidin-coated MNPs can only capture approximately $0.01 \mu \mathrm{M}$ of biotin groups. Due to small size and short diffusion time, free biotinylated inner primers as well as short biotinylated amplicons would preferentially bind to the streptavidin-coated MNPs in the labeling step, leading to a blocking effect that prevents the binding of long amplicons to MNPs. To investigate this hypothesis, the hydrodynamic size distribution of ampliconbound MNPs (1 pM target sequence) was measured by DLS where the blank control sample represents MNPs reacted with negative LAMP results, i.e., primer-blocked MNPs. Based on the average hydrodynamic diameters of amplicon-MNPs obtained from the size distributions in Figure 3a (from left to right: 259.9, 436.6, 502.4, and $566.2 \mathrm{~nm}$ ), the mean lengths of the amplicons could be estimated under the simplifying assumptions of a uniform coverage of the MNPs and that the amplicons were rigid and with a length of $0.34 \mathrm{~nm} / \mathrm{bp} .{ }^{45}$ For 
a)

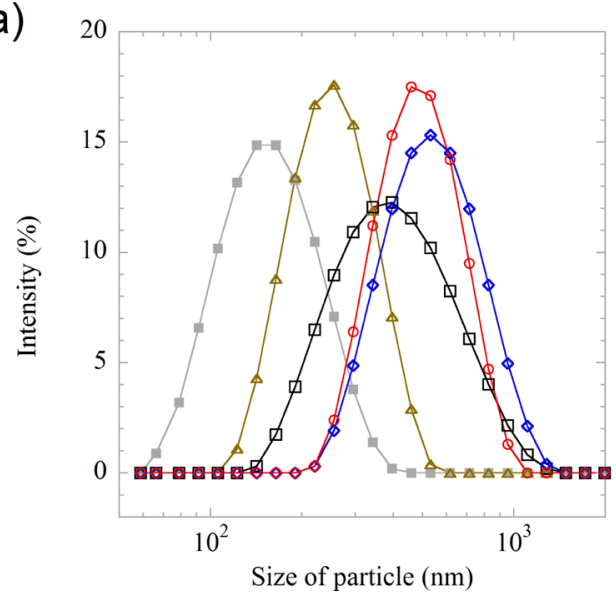

b)

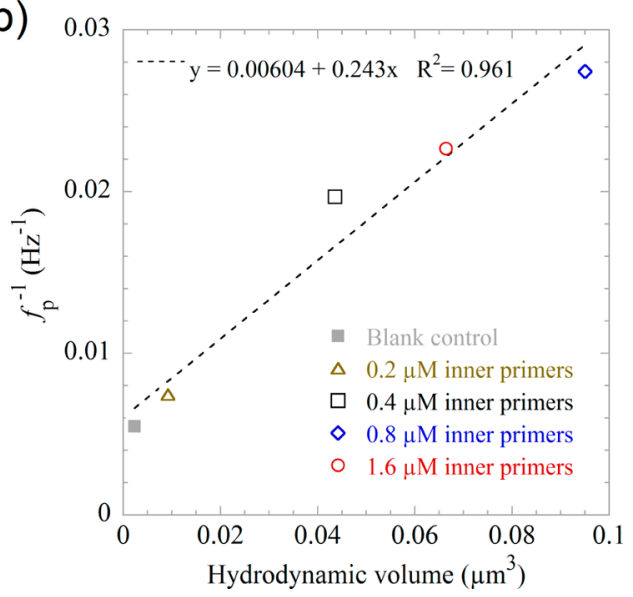

Figure 3. Results obtained from dynamic light scattering and the comparison with that of optomagnetic measurement. A volume of $20 \mu \mathrm{L}$ of synthetic DNA was used for each measurement. (a) Hydrodynamic size distribution of amplicon-MNPs formed using the indicated inner primer concentrations. The blank control sample (gray solid squares) represents MNPs reacted with negative LAMP results, i.e., primer-blocked MNPs. (b) Inverse peak frequency of optomagnetic spectra in Figure $2 \mathrm{~b}$ vs mean hydrodynamic volume calculated from DLS results. A linear relationship is observed $\left(R^{2}>0.96\right)$.

inner primer concentrations of $0.2,0.4,0.8$, and $1.6 \mu \mathrm{M}$, the resulting mean lengths of MNP-bound amplicons were 88, 348, 539 , and 445 base pairs, respectively. It should be noted that this calculation is approximate since the amplicons are flexible and also because single-stranded biotinylated primers bind to the MNPs. The mean MNP-bound amplicon lengths are marked in Figure S3b by red arrows, and by comparing the arrow positions on the gel electrophoresis photograph it can indeed be concluded that rather small amplicons are preferentially bound to the MNPs. Furthermore, in Figure $3 \mathrm{~b}$, the mean hydrodynamic volumes calculated from the DLS results are plotted against $1 / f_{\mathrm{p}}$ obtained from Figure $2 \mathrm{a}$ and Figure S5. A linear correlation $\left(R^{2}>0.96\right)$ is observed between $1 / f_{\mathrm{p}}$ and the mean hydrodynamic size (obtained from DLS). This strongly supports our hypothesis that there is a gradual hydrodynamic size increase of individual MNPs (Figure 3a) upon increasing amounts of LAMP products (Figure S3b). In addition, DLS results also show that the inner primer concentration of $0.8 \mu \mathrm{M}$ (blue diamonds in Figure 3) provided the largest change in the hydrodynamic volume of MNPs (increased from 0.002 to $0.095 \mu \mathrm{m}^{3}$ ). Considered the optimum value, a $0.8 \mu \mathrm{M}$ concentration of inner primers was employed in the subsequent study on clinical samples.

Representative reported LAMP-based biosensors are summarized in Table 1 with LODs and total assay times. Among the LAMP-based biosensors, the optomagnetic readout system is competitive in both device cost and running cost. In addition, the optomagnetic sensor has the potential to enhance the accuracy of detection and reduce the risk of contamination since there is no need to immobilize the target molecules on a chip/electrode or remove unbound target molecules prior to quantification. Further, the optomagnetic readout system is compatible with any disposable and low-cost transparent plastic sample container.

Detection of NDV RNA from Tissue Specimens. To evaluate the applicability of the proposed approach for detection of clinical samples, we tested whether the LAMPOptomag system could be applied for the detection of viral RNA extracted from tissues and vaccine. Twenty-nine clinical specimens were measured by both real-time RT-PCR and our approach, as shown in Figure 4 (negative control results not

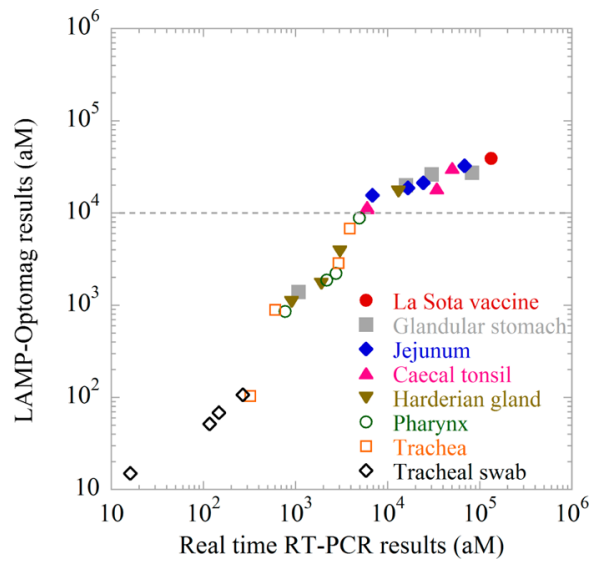

Figure 4. Analyses of clinical NDV specimens (vaccine and tissue) by real-time RT-PCR ( $x$-axis) and the LAMP-Optomag measurement $(y$ axis). A volume of $20 \mu \mathrm{L}$ extracts of clinical specimens were used and the measurements were repeated just once (only for qualitative comparison of the two approaches). The upper limit of the dynamic detection range of LAMP-Optomag $\left(10^{4} \mathrm{aM}\right)$ is shown as a horizontal dashed line. A linear relationship is observed $\left(R^{2}>0.93\right)$ within the dynamic detection range of $10-10^{4} \mathrm{aM}$.

shown). No false-positive or false-negative results can be observed. Within the dynamic detection range of the LAMPOptomag system, the quantitative results were consistent with real time RT-PCR results. It should be noted that the quantification of clinical samples can be further optimized since the dose-response curve used here for quantification (see Figure S9, the dynamic detection range is $10-10^{4} \mathrm{aM}$ ) was obtained from detection of synthetic target DNA. According to the real-time RT-PCR results, the lowest concentration of the positive specimen (from tracheal swab) was 9.5 copies $/ \mu \mathrm{L}$ (close to the LOD of real-time RT-PCR method), which can also be detected by the LAMP-Optomag system. These results demonstrate that our approach is highly robust for clinical samples and can achieve an LOD similar to the real-time RTPCR method.

Although the proposed LAMP-Optomag system can achieve an ultrasensitive detection within $30 \mathrm{~min}$ without the use of 
sophisticated thermal cycling devices (while the total assay time for real-time RT-PCR based NDV detection is $80 \mathrm{~min}$ or more), ${ }^{41-44}$ its dynamic detection range is narrower than realtime RT-PCR. As shown in Figure 4, the signal was saturated for nearly half of the investigated clinical specimens. For studies where quantification is needed, this issue can be solved by sample dilution. Finally, it should be stressed that although a saturation level in the dose-response curve is reached for target concentrations above $10^{4} \mathrm{aM}$, a target concentration above $10^{4}$ $\mathrm{aM}$ will not be misinterpreted as a lower concentration.

Despite the consistency of the output signal from our assay with results from DLS and real-time RT-PCR, complicating factors exist in the current detection format which limit deeper understanding of the mechanisms behind the assay and which lower the quantification accuracy: (1) the amplification efficiency of LAMP for an individual target is difficult to predict; (2) the size distribution of LAMP amplicons is not straightforward to analyze; (3) the probability of biotinylated amplicons to be captured by MNPs depends on their size; and (4) there is a competition between biotinylated amplicons and free biotinylated primers to be captured by MNPs. Further studies, outside the scope of this paper, are needed to optimize the experimental design in order to model the assay in detail.

\section{CONCLUSIONS}

We have demonstrated a rapid, ultrasensitive and cost-efficient DNA/RNA detection strategy using LAMP and RT-LAMP in combination with an optomagnetic sensor readout. We employed this method to detect a synthetic target DNA sequence (corresponding to a target gene for NDV strain Herts/33) and achieved an LOD of $10 \mathrm{aM}$ with a total assay time of $30 \mathrm{~min}$. The method was validated by detection of clinical NDV RNA samples. Using LAMP eliminates the need for thermal cycling devices, and can provide high amplification efficiency using a simple one-step protocol. The $V_{2}^{\prime} / V_{0}$ peak frequency calculation method that is used to quantify the target concentration is very convenient since it is not noticeably influenced by slight sample-to-sample variations in magnetic nanoparticle content, presence of LAMP byproducts, fluctuations of the laser intensity and slight variations of transmission properties of the cuvettes. The performance of our approach using clinical specimens is comparable with real-time RT$\mathrm{PCR}^{41-44}$ and is about 100 times more sensitive than turbiditybased naked eye judgment, implying that the proposed LAMPOptomag method is robust and reliable. In addition, by changing the LAMP primers, this strategy can serve as a general method for the detection of other DNA/RNA sequences with high specificity and sensitivity. Finally, it should be emphasized that we have used end-point detection in the current study but it is rather straightforward to monitor the peak position in realtime. A fully integrated one-step system with real-time read-out and automated sample preparation can be accomplished by using a spinning microfluidic disc version of our optomagnetic system, ${ }^{34}$ which can quantify target concentrations above the current upper limit of the dynamic range without reducing the sensitivity.

\section{ASSOCIATED CONTENT}

\section{S Supporting Information}

The Supporting Information is available free of charge on the ACS Publications website at DOI: 10.1021/acssensors.6b00379.
Description of the optomagnetic system and measurement principle, table of oligonucleotides used in this study, agarose gel electrophoresis analysis results, SEM micrographs of the $\mathrm{Mg}_{2} \mathrm{P}_{2} \mathrm{O}_{7}$ precipitation, and data of the optimization (PDF)

Primer corresponding position (XLSX)

\section{AUTHOR INFORMATION}

\section{Corresponding Author}

*E-mail: mattias.stromberg@angstrom.uu.se.

\section{Notes}

The authors declare no competing financial interest.

\section{ACKNOWLEDGMENTS}

This research was financially supported by Swedish Research Council Formas (project numbers 221-2012-444, 221-2014574, and 2011-1692). Zhen Qiu at Department of Engineering Sciences, Uppsala University, is gratefully acknowledged for helpful assistance when running the scanning electron microscope.

\section{REFERENCES}

(1) Daszak, P.; Cunningham, A. A.; Hyatt, A. D. Wildlife ecology Emerging infectious diseases of wildlife - Threats to biodiversity and human health. Science 2000, 287, 443-449.

(2) Aldous, E. W.; Alexander, D. J. Detection and differentiation of Newcastle disease virus (avian paramyxovirus type 1). Avian Pathol. 2001, 30, 117-128.

(3) Alexander, D. J.; Aldous, E. W.; Fuller, C. M. The long view: a selective review of 40 years of Newcastle disease research. Avian Pathol. 2012, 41, 329-335.

(4) Ganar, K.; Das, M.; Sinha, S.; Kumar, S. Newcastle disease virus: Current status and our understanding. Virus Res. 2014, 184, 71-81.

(5) Chaka, H.; Thompson, P. N.; Goutard, F.; Grosbois, V. Evaluation of enzyme-linked immunosorbent assays and a haemagglutination inhibition tests for the detection of antibodies to Newcastle disease virus in village chickens using a Bayesian approach. Prev. Vet. Med. 2015, 119, 21-30.

(6) Liu, L.; Benyeda, Z.; Zohari, S.; Yacoub, A.; Isaksson, M.; Leijon, M.; LeBlanc, N.; Benyeda, J.; Belak, S. Assessment of Preparation of Samples Under the Field Conditions and a Portable Real-Time RTPCR Assay for the Rapid On-Site Detection of Newcastle Disease Virus. Transboundary Emerging Dis. 2016, 63, e245-250.

(7) Notomi, T.; Okayama, H.; Masubuchi, H.; Yonekawa, T.; Watanabe, K.; Amino, N.; Hase, T. Loop-mediated isothermal amplification of DNA. Nucleic Acids Res. 2000, 28, e63.

(8) Tomita, N.; Mori, Y.; Kanda, H.; Notomi, T. Loop-mediated isothermal amplification (LAMP) of gene sequences and simple visual detection of products. Nat. Protoc. 2008, 3 (5), 877-882.

(9) Parida, M.; Sannarangaiah, S.; Dash, P. K.; Rao, P. V. L; Morita, $\mathrm{K}$. Loop mediated isothermal amplification (LAMP): a new generation of innovative gene amplification technique; perspectives in clinical diagnosis of infectious diseases. Rev. Med. Virol. 2008, 18 (18), 407421 .

(10) Zhang, X. Z.; Lowe, S. B.; Gooding, J. J. Brief review of monitoring methods for loop-mediated isothermal amplification (LAMP). Biosens. Bioelectron. 2014, 61, 491-499.

(11) Mori, Y.; Nagamine, K.; Tomita, N.; Notomi, T. Detection of loop-mediated isothermal amplification reaction by turbidity derived from magnesium pyrophosphate formation. Biochem. Biophys. Res. Commun. 2001, 289, 150-154.

(12) Le, T. H.; Nguyen, N. T. B.; Truong, N. H.; De, N. V. Development of Mitochondrial Loop-Mediated Isothermal Amplification for Detection of the Small Liver Fluke Opisthorchis viverrini (Opisthorchiidae; Trematoda; Platyhelminthes). J. Clin. Microbiol. 2012, 50, 1178-1184. 
(13) Sun, W.; Qin, P.; Gao, H. W.; Li, G. C.; Jiao, K. Electrochemical DNA biosensor based on chitosan/nano-V2O5/MWCNTs composite film modified carbon ionic liquid electrode and its application to the LAMP product of Yersinia enterocolitica gene sequence. Biosens. Bioelectron. 2010, 25, 1264-1270.

(14) Hsieh, K. W.; Patterson, A. S.; Ferguson, B. S.; Plaxco, K. W.; Soh, H. T. Rapid, Sensitive, and Quantitative Detection of Pathogenic DNA at the Point of Care through Microfluidic Electrochemical Quantitative Loop-Mediated Isothermal Amplification. Angew. Chem., Int. Ed. 2012, 51, 4896-4900.

(15) Tlili, C.; Sokullu, E.; Safavieh, M.; Tolba, M.; Ahmed, M. U.; Zourob, M. Bacteria Screening, Viability, And Confirmation Assays Using Bacteriophage-Impedimetric/Loop-Mediated Isothermal Amplification Dual-Response Biosensors. Anal. Chem. 2013, 85, 4893-4901. (16) Xie, S. B.; Yuan, Y. L.; Chai, Y. Q.; Yuan, R. Tracing Phosphate Ions Generated during Loop-Mediated Isothermal Amplification for Electrochemical Detection of Nosema bombycis Genomic DNA PTP1. Anal. Chem. 2015, 87, 10268-10274.

(17) Chuang, T. L.; Wei, S. C.; Lee, S. Y.; Lin, C. W. A polycarbonate based surface plasmon resonance sensing cartridge for high sensitivity HBV loop-mediated isothermal amplification. Biosens. Bioelectron. 2012, 32, 89-95.

(18) Nawattanapaiboon, K.; Kiatpathomchai, W.; Santanirand, P.; Vongsakulyanon, A.; Amarit, R.; Somboonkaew, A.; Sutapun, B.; Srikhirin, T. SPR-DNA array for detection of methicillin-resistant Staphylococcus aureus (MRSA) in combination with loop-mediated isothermal amplification. Biosens. Bioelectron. 2015, 74, 335-340.

(19) Yang, A. K. L.; Lu, H. F.; Wu, S. Y.; Kwok, H. C.; Ho, H. P.; Yu, S.; Cheung, A. K. L.; Kong, S. K. Detection of Panton-Valentine Leukocidin DNA from methicillin-resistant Staphylococcus aureus by resistive pulse sensing and loop-mediated isothermal amplification with gold nanoparticles. Anal. Chim. Acta 2013, 782, 46-53.

(20) Prakrankamanant, P.; Leelayuwat, C.; Promptmas, C.; Limpaiboon, T.; Wanram, S.; Prasongdee, P.; Pientong, C.; Daduang, J.; Jearanaikoon, P. The development of DNA-based quartz crystal microbalance integrated with isothermal DNA amplification system for human papillomavirus type 58 detection. Biosens. Bioelectron. 2013, 40, 252-257.

(21) Veigas, B.; Branquinho, R.; Pinto, J. V.; Wojcik, P. J.; Martins, R.; Fortunato, E.; Baptista, P. V. Ion sensing (EIS) real-time quantitative monitorization of isothermal DNA amplification. Biosens. Bioelectron. 2014, 52, 50-55.

(22) Zhi, X.; Deng, M.; Yang, H.; Gao, G.; Wang, K.; Fu, H. L.; Zhang, Y. X.; Chen, D.; Cui, D. X. A novel HBV genotypes detecting system combined with microfluidic chip, loop-mediated isothermal amplification and GMR sensors. Biosens. Bioelectron. 2014, 54, 372377.

(23) Draz, M. S.; Lu, X. Development of a Loop Mediated Isothermal Amplification (LAMP) - Surface Enhanced Raman spectroscopy (SERS) Assay for the Detection of Salmonella Enterica Serotype Enteritidis. Theranostics 2016, 6, 522-532.

(24) Kelley, S. O.; Mirkin, C. A.; Walt, D. R.; Ismagilov, R. F.; Toner, M.; Sargent, E. H. Advancing the speed, sensitivity and accuracy of biomolecular detection using multi-length-scale engineering. Nat. Nanotechnol. 2014, 9, 969-980.

(25) Lee, H.; Shin, T. H.; Cheon, J.; Weissleder, R. Recent Developments in Magnetic Diagnostic Systems. Chem. Rev. 2015, 115, 10690-10724.

(26) Park, S. Y.; Handa, H.; Sandhu, A. Magneto-optical biosensing platform based on light scattering from self-assembled chains of functionalized rotating magnetic beads. Nano Lett. 2010, 10, 446-451.

(27) Ranzoni, A.; Schleipen, J. J.; van Ijzendoorn, L. J.; Prins, M. W. Frequency-selective rotation of two-particle nanoactuators for rapid and sensitive detection of biomolecules. Nano Lett. 2011, 11, 20172022.

(28) Ranzoni, A.; Sabatte, G.; van Ijzendoorn, L. J.; Prins, M. W. One-step homogeneous magnetic nanoparticle immunoassay for biomarker detection directly in blood plasma. ACS Nano 2012, 6, 3134-3141.
(29) Ramiandrisoa, D.; Brient-Litzler, E.; Daynes, A.; Compain, E.; Bibette, J.; Baudry, J. Optical protein detection based on magnetic clusters rotation. New Biotechnol. 2015, 32, 467-472.

(30) Donolato, M.; Antunes, P.; Bejhed, R. S.; Zardán Gómez de la Torre, T.; Østerberg, F. W.; Strömberg, M.; Nilsson, M.; Strømme, M.; Svedlindh, P.; Hansen, M. F.; Vavassori, P. Novel Readout Method for Molecular Diagnostic Assays Based on Optical Measurements of Magnetic Nanobead Dynamics. Anal. Chem. 2015, 87, 1622-1629.

(31) Yang, J.; Donolato, M.; Pinto, A.; Bosco, F. G.; Hwu, E. T.; Chen, C. H.; Alstrom, T. S.; Lee, G. H.; Schafer, T.; Vavassori, P.; Boisen, A.; Lin, Q.; Hansen, M. F. Blu-ray based optomagnetic aptasensor for detection of small molecules. Biosens. Bioelectron. 2016, $75,396-403$.

(32) Tian, B.; Bejhed, R. S.; Svedlindh, P.; Strömberg, M. Blu-ray optomagnetic measurement based competitive immunoassay for Salmonella detection. Biosens. Bioelectron. 2016, 77, 32-39.

(33) Antunes, P.; Watterson, D.; Parmvi, M.; Burger, R.; Boisen, A.; Young, P.; Cooper, M. A.; Hansen, M. F.; Ranzoni, A.; Donolato, M. Quantification of NS1 dengue biomarker in serum via optomagnetic nanocluster detection. Sci. Rep. 2015, 5, 16145.

(34) Donolato, M.; Antunes, P.; Zardán Gómez de la Torre, T.; Hwu, E. T.; Chen, C. H.; Burger, R.; Rizzi, G.; Bosco, F. G.; Strømme, M.; Boisen, A.; Hansen, M. F. Quantification of rolling circle amplified DNA using magnetic nanobeads and a Blu-ray optical pick-up unit. Biosens. Bioelectron. 2015, 67, 649-655.

(35) Mezger, A.; Fock, J.; Antunes, P.; Østerberg, F. W.; Boisen, A.; Nilsson, M.; Hansen, M. F.; Ahlford, A.; Donolato, M. Scalable DNABased Magnetic Nanoparticle Agglutination Assay for Bacterial Detection in Patient Samples. ACS Nano 2015, 9, 7374-7382.

(36) Pham, H. M.; Nakajima, C.; Ohashi, K.; Onuma, M. Loopmediated isothermal amplification for rapid detection of Newcastle disease virus. J. Clin. Microbiol. 2005, 43, 1646-1650.

(37) Li, Q.; Xue, C. Y.; Qin, J. P.; Zhou, Q. F.; Chen, F.; Bi, Y. Z.; Cao, Y. C. An improved reverse transcription loop-mediated isothermal amplification assay for sensitive and specific detection of Newcastle disease virus. Arch. Virol. 2009, 154, 1433-1440.

(38) Strömberg, M.; Göransson, J.; Gunnarsson, K.; Nilsson, M.; Svedlindh, P.; Strømme, M. Sensitive molecular diagnostics using volume-amplified magnetic nanobeads. Nano Lett. 2008, 8, 816-821.

(39) Zardán Gómez de la Torre, T.; Ke, R.; Mezger, A.; Svedlindh, P.; Strømme, M.; Nilsson, M. Sensitive Detection of Spores Using Volume-Amplified Magnetic Nanobeads. Small 2012, 8, 2174-2177.

(40) Østerberg, F. W.; Rizzi, G.; Donolato, M.; Bejhed, R. S.; Mezger, A.; Strömberg, M.; Nilsson, M.; Strømme, M.; Svedlindh, P.; Hansen, M. F. On-Chip Detection of Rolling Circle Amplified DNA Molecules from Bacillus Globigii Spores and Vibrio Cholerae. Small 2014, 10, 2877-2882.

(41) Wise, M. G.; Suarez, D. L.; Seal, B. S.; Pedersen, J. C.; Senne, D. A.; King, D. J.; Kapczynski, D. R.; Spackman, E. Development of a real-time reverse-transcription PCR for detection of Newcastle disease virus RNA in clinical samples. J. Clin. Microbiol. 2004, 42, 329-338.

(42) Antal, M.; Farkas, T.; German, P.; Belak, S.; Kiss, I. Real-time reverse transcription-polymerase chain reaction detection of Newcastle disease virus using light upon extension fluorogenic primers. J. Vet. Diagn. Invest. 2007, 19, 400-404.

(43) Kim, L. M.; King, D. J.; Suarez, D. L.; Wong, C. W.; Afonso, C. L. Characterization of class I Newcastle disease virus isolates from Hong kong live bird markets and detection using real-time reverse transcription-PCR. J. Clin. Microbiol. 2007, 45, 1310-1314.

(44) Nidzworski, D.; Rabalski, L.; Gromadzka, B. Detection and differentiation of virulent and avirulent strains of Newcastle disease virus by real-time PCR. J. Virol. Methods 2011, 173, 144-149.

(45) Marko, J. F.; Siggia, E. D. Stretching DNA. Macromolecules 1995, 28, 8759-8770. 